\title{
Avaliação de crianças com indicação de dificuldades de aprendizagem pela bateria Woodcock-Johnson III
}

\author{
Avaliação das dificuldades de aprendizagem
}

\author{
Dalva Alice Rocha Mól \\ Solange Muglia Wechsler
}

\section{Resumo}

Este estudo investigou as habilidades cognitivas de crianças com indicação de dificuldades de aprendizagem por seus professores. Participaram 60 crianças de ambos os sexos, idades de 7 a 10 anos, 30 sem indicação de dificuldades de aprendizagem e 30 com indicação de dificuldades de aprendizagem da primeira e segunda série, e oito professores. Utilizou-se a Bateria de Habilidades Cognitivas Woodcock-Johnson III (WJ III), composta por 10 testes e as notas escolares em Português, Matemática e Ciências. A Análise da Variância apontou efeitos de grupo em todas as habilidades avaliadas de série escolar. A correlação de Pearson apontou relações significativas entre as habilidades inteligência cristalizada, recuperação à longo prazo, processamento auditivo, raciocínio fluido e memória de trabalho com as notas de Português, como também desta última com a Matemática. Concluiu-se que a WJ III é válida para identificar dificuldades de aprendizagem.

Palavras-chave: Dificuldades de aprendizagem, Bateria Woodcock-Johnson III, Validação.

\section{Assessment of children referred as learning disabled with the Woodcock- Johnson III}

\begin{abstract}
This study investigates the cognitive abilities of children referred as having learning difficulties by their teachers. The participants were 60 children of both sexes, 30 with difficulties and 30 without difficulties, ages ranging 7-10 years old, attending $1^{\text {st }}$ and $2^{\text {nd }}$ elementary grades in public schools in Sao Paulo state, as well as their teachers. The instruments were: the Woodcock-Johnson III Tests of Cognitive Abilities (WJ-III) composed of 10 tests, and school grades in Portuguese, Math and Science. The results from Multivariate and Univariate Analysis of Variance indicated significant group differences for all cognitive abilities measured. Pearson correlations demonstrated significant relationships among abilities related to crystallized intelligence, long term retrieval, auditory processing, fluid reasoning, and working memory with grades in Portuguese, whereas the last one was also significant related to Mathematics. In conclusion, the Woodcock Johnson III Battery was confirmed as a valid measure to identify learning difficulties among Brazilian children.

Keywords: Learning disabilities, Woodcock-Johnson Psychoeducational Battery, Validation.
\end{abstract}

\section{Evaluación de niños con indicativos de dificultades de aprendizaje por la batería Woodcock-Johnson III}

\footnotetext{
Resumen

Este estudio investigó las habilidades cognitivas de niños con indicativos de dificultades de aprendizaje de acuerdo con sus profesores. Participaron 60 niños de ambos sexos, edad de 7 a 10 años, 30 sin indicativo de dificultades de aprendizaje y 30 con indicativo de dificultades de aprendizaje del primero y segundo grado, y ocho profesores. Se utilizó la Batería de Habilidades Cognitivas Woodcock-Johnson III (WJ III), compuesta por 10 testes y las notas escolares de Portugués, Matemáticas y Ciencias. El Análisis de Variancia apuntó efectos de grupo en todas las habilidades evaluadas del grado escolar. La correlación de Pearson apuntó relaciones significativas entre las habilidades inteligencia cristalizada, recuperación a largo plazo, procesamiento auditivo, raciocinio fluido y memoria de trabajo con las notas de Portugués, así como también de esta última con Matemáticas. Se concluyó que la WJ III es válida para identificar dificultades de aprendizaje.

Palabras clave: Dificultades de aprendizaje, Batería Woodcock-Johnson III, Validación.
} 


\section{Introdução}

Durante o processo de escolarização a criança tem acesso ao conhecimento formal. Além de proporcionar condições para que a criança apreenda esse conhecimento, a escola deve se tornar um espaço de aprendizagem que permita à criança expandir e desenvolver novas formas de relações com o mundo.

No entanto, ao longo do processo de escolarização a criança pode enfrentar vários obstáculos. Um grande contingente de alunos apresenta dificuldades de aprendizagem nas séries iniciais. Essas crianças, na maioria das vezes, são tratadas pelos professores na escola de forma preconceituosa e são discriminadas, sem que se investiguem suas reais habilidades e potencialidades (Ciasca, 2003; Corsini, 1998).

Muitas vezes as crianças que apresentam dificuldades na aprendizagem escolar são encaminhadas aos serviços de saúde como se os problemas escolares fossem problemas de saúde (Boarini \& Borges, 1998; Rossini \& Santos, 2002). No entanto, pode-se dizer que 0 desempenho escolar é influenciado por múltiplos fatores e, muitas vezes, a escola torna-se ineficaz para lidar com a diversidade dos alunos. Diante disso, torna-se necessário discutir o conceito de dificuldades de aprendizagem e sua relação com o desempenho acadêmico, pois um dos fatores relacionados ao rendimento escolar são as dificuldades de aprendizagem do aluno.

As dificuldades de aprendizagem foram e são identificadas por diferentes critérios, fato que contribuiu para a disseminação de várias definições. Na concepção educacional, as dificuldades de aprendizagem relacionamse à habilidade imperfeita de escutar, pensar, ler, escrever, soletrar e de fazer cálculos. Nessa definição são excluídas crianças que tenham deficiência sensorial ou deficiência mental (Dombrowski, Reynolds \& Kamphaus, 2004; Sisto, 2002).

Vale comentar que há necessidade em distinguir dificuldades de aprendizagem e distúrbios de aprendizagem. Os distúrbios de aprendizagem relacionamse a um quadro em que várias capacidades da criança estão no limite da normalidade. No entanto, a criança não consegue aprender de forma global ou específica, há deficiência em seus processos perceptivos, de integração ou expressão (Azevedo, 2002). Essa concepção deixa claro que, os distúrbios de aprendizagem têm componentes que indicam algum tipo de disfunção do sistema nervoso central que está impedindo o rendimento acadêmico da criança, mesmo que o potencial intelectual permita o alcance de novas aprendizagens.

As pesquisas sobre as dificuldades de aprendizagem são diversificadas. $\mathrm{Na}$ revisão brasileira, os trabalhos encontram-se na interface da Psicologia e da Educação. Convém comentar que, nos estudos pesquisados, encontrou-se a discussão sobre o conceito de dificuldade de aprendizagem e as limitações que a ambigüidade conceitual pode acarretar na identificação, diagnóstico e modelos de intervenção neste campo. As pesquisas sobre dificuldades de aprendizagem aparecem associadas a temas como interações sociais (Saravali, 2003), dificuldades específicas da leitura e/ou da escrita (Bispo, 2000; Carneiro, 2002; Coll, 2000; Zucoloto, 2001; Zucoloto \& Sisto, 2002), a atuação do psicólogo nesta área e os tipos de intervenções que estão sendo oferecidas para crianças que apresentem dificuldades na escola (Azevedo, 2002; Cabral \& Sawaya, 2001). De modo geral, os trabalhos desenvolvidos associam-se à questão do fracasso escolar e à tentativa de encontrar soluções que permitam encontrar novas estratégias para melhorar as condições de aprendizagem na escola.

Quanto à avaliação das habilidades cognitivas em crianças com dificuldades de aprendizagem, os estudos realizados relacionam-se à identificação de habilidades cognitivas associadas ao desempenho escolar (Capovilla, Güstchow \& Capovilla, 2004; Kaminski \& Good, 1996; Kennard, Stewart, Silver \& Emillie, 2000; Stanford \& Oakland, 2000). Esses autores utilizaram instrumentos que avaliam habilidades como: raciocínio abstrato, atenção e concentração, raciocínio não-verbal, consciência fonológica, memória fonológica, considerando que estas são habilidades específicas e necessárias ao sucesso acadêmico.

Nesse contexto desenvolveu-se um projeto para avaliar as habilidades cognitivas de crianças com dificuldades de aprendizagem, no sentido de demonstrar que as habilidades cognitivas representam um dos vários fatores que interferem no desempenho acadêmico. O conhecimento das características cognitivas das crianças possibilita ao professor a aplicação de novas estratégias de ensino. Dessa forma, optou-se pela utilização da Bateria de Habilidades Cognitivas Woodcock-Johnson III apoiada no modelo de Cattell-Horn-Carroll (CHC) das capacidades cognitivas (McGrew \& Flanagan, 1998; Flanagan, McGrew \& Ortiz, 2000).

O modelo Catell-Horn-Carrol foi desenvolvido por McGrew e Flanagan (1998) com o objetivo de atualizar a prática da avaliação de acordo com a teoria psicométrica contemporânea. Segundo Flanagan e Ortiz (2001) há várias tentativas de analisar e classificar as habilidades cognitivas humanas. A proposta teórica do modelo $\mathrm{CHC}$ contempla a integração da teoria Gf-Gc de Cattell-Horn e Carrol. Esse modelo enfatiza a camada II e I de Carroll e especifica dez habilidades gerais das capacidades cognitivas (Santos \& Primi, 2005). As habilidades gerais que compõem o modelo $\mathrm{CHC}$ são elencadas por Flanagan e Ortiz (2001): Inteligência fluida (Gf), Inteligência Cristalizada (Gc), Conhecimento Quantitativo (Gq), Habilidade de leitura e escrita (Gwr), Memória a curto prazo (Gsm), Processamento visual (Gv), Processamento auditivo (Ga), Recuperação de memória de longo prazo (Glr), Velocidade de processamento (Gs).

$\mathrm{O}$ modelo $\mathrm{CHC}$ tem motivado investigações relacionadas à construção e validação de novos instrumentos psicológicos para avaliar as diferentes habilidades cognitivas. A Bateria de Habilidades Cognitivas Woodcock-Johnson III é um instrumento construído com aporte teórico no modelo CHC (Muñoz-Sandoval \& Woodcock, 1999; Woodcock-johnson, McGrew \& Mather, 2001) e permite identificar habilidades cognitivas que têm relevância para o desempenho em tarefas escolares.

As pesquisas realizadas, no Brasil, com a Bateria Woodcock-Johnson III relacionam a estudos de adaptação e validação (Wechsler, Vendramini \& Schelini, no prelo; Wechsler \& Schelini, 2006). Os resultados dessas pesquisas têm apontado que a WJ III é uma medida válida para a população brasileira e poderá possibilitar um melhor diagnóstico das dificuldades de crianças e jovens, principalmente aquelas relacionadas à alfabetização e matemática. 
Desse modo, o presente estudo teve como objetivo principal avaliar as habilidades cognitivas de crianças com indicação de dificuldades de aprendizagem pela Bateria Woodcock-Johnson III.

\section{Método}

\section{Participantes}

Os participantes desta pesquisa foram 60 crianças, que freqüentavam a primeira e segunda série do Ensino Fundamental de duas escolas públicas, sendo 30 crianças sem indicação de dificuldades de aprendizagem e 30 crianças com dificuldades de aprendizagem. A amostra total foi composta 31 alunos do sexo masculino $(51,7 \%)$, $55 \%$ freqüentavam a segunda série. $O$ grupo sem dificuldade foi indicado pelo professor utilizando o critério de que não necessitavam do reforço escolar. Neste grupo $62,1 \%$ foi composto por meninas e $60,6 \%$ das crianças representavam a segunda série, a faixa etária predominante foi de oito anos (50\%). No grupo com dificuldade $61,3 \%$ eram crianças do sexo masculino, sendo $63 \%$ alunos da primeira série. Neste grupo $43,3 \%$ tinham sete anos. Os alunos com dificuldades freqüentavam 0 reforço escolar.

\section{Instrumentos}

Os instrumentos utilizados para coletar os dados foram compostos pela Bateria de Habilidades Cognitivas Woodcock-Johnson III, composta por dez testes originais e que abrangem as seguintes áreas:

Teste 1: Inteligência Cristalizada (Gc), composto pelo subteste Compreensão verbal e Conhecimento: Vocabulário ( $1 \mathrm{~A}=70$ itens), Sinônimos ( $1 \mathrm{~B}=42$ itens), Antônimos ( $1 \mathrm{C}=40$ itens) e Analogias ( $1 \mathrm{D}=40$ itens). Total $=192$ itens

Teste 2: Recuperação a longo prazo (Glr). Este teste é representado pelo subteste Aprendizagem visual auditiva ( $2=7$ itens $)$.

Teste 3: Pensamento Viso-Espacial (Gv), representado pelo subteste Relações Espaciais $(3=33$ conjunto de itens).

Teste 4: Processamento auditivo (Ga). Esse teste é representado pelo subteste Combinação de Sons $(4=33$ itens).

Teste 5: O Raciocínio Fluido (Gf) composto pelo subteste Formação de Conceitos ( $5=60$ séries de itens).

Teste 6: Rapidez de processamento (Gs), composto pelo subteste Emparelhamento Visual $(6=60$ séries de itens).

Teste 7: Memória de trabalho (Gsm), presente no subteste Números invertidos ( $7=30$ itens).

Teste 8: Processamento Auditivo (Ga), representado pelo subteste Palavras Incompletas ( $8=44$ itens).

Teste 9: Memória auditiva (Gsm). Composto pelo subteste Memória Auditiva (9P e 9N = 21 itens).

Teste 10: Recuperação a longo prazo (Glr), representado pelo subteste Aprendizagem visual adiada (10 $=25$ itens).

2) Fichas com aproveitamento escolar dos alunos, com as notas escolares do segundo bimestre. $O$ aproveitamento escolar é apresentado na forma de conceitos e correspondem, em termos de notas, da seguinte maneira: $A=9-10, B=7-8, C=5-6, D=4$.

\section{Procedimento}

Após permissão do Comitê de Ética Institucional estabeleceu-se o primeiro contato com a direção das escolas, sendo expostos os objetivos da pesquisa. Para solicitar autorização utilizou-se uma carta de ciência e autorização para realização da pesquisa nas duas instituições e um termo de consentimento livre e esclarecido para os pais das crianças.

A pesquisa junto aos alunos consistiu na aplicação da Bateria de Habilidades cognitivas Woodcock-Johnson III, que foi realizada durante três sessões individuais de uma hora aproximadamente. Foram aplicados todos os itens da versão original e da versão brasileira adaptada por Wechsler e Schelini (2006). As análises foram realizadas considerando os resultados brutos. A ordem de aplicação dos testes ocorreu da seguinte maneira: na primeira sessão foram aplicados os testes de compreensão verbal; sinônimos; antônimos; analogias verbais; aprendizagem visual auditiva e relações espaciais. A segunda sessão foi aplicada após intervalo de uma semana, o espaço entre a primeira e a segunda sessão foi o mesmo para todos os participantes, pois era necessário devido ao teste de aprendizagem visual auditiva adiada. Os testes da segunda sessão foram: aprendizagem visual auditiva adiada; formação de conceitos; combinação visual e números invertidos. Na terceira sessão foram aplicados os testes de combinação de sons; palavra incompleta e memória auditiva. Esses testes foram aplicados com a utilização de uma fita cassete.

O aproveitamento escolar dos alunos foi fornecido pela secretaria das escolas, que mostraram os conceitos e como as notas eram convertidas em conceitos. Nas escolas estudadas o sistema de avaliação realiza-se da seguinte maneira: seis pontos são distribuídos em provas dos conteúdos curriculares, dois pontos em atividades de sala de aula e dois pontos referem às tarefas que os alunos realizam. A conversão dos conceitos em notas foi realizada utilizando o critério do valor máximo para cada conceito.

Após a coleta dos dados procedeu-se a sua análise a fim de estudar os efeitos de grupo, sexo e série. A idade foi mantida como co-variável. Primeiramente utilizou-se a análise multivariada, e posteriormente, a análise de variância. Os resultados da WJ-III foram correlacionados com as notas escolares dos alunos.

\section{Resultados}

Inicialmente, apresentar-se-ão as médias e desviospadrão das habilidades cognitivas da Bateria WoodcockJohnson III considerando a variável série escolar, para os grupos sem e com dificuldades de aprendizagem.

$\mathrm{Na}$ Tabela 2, os dados apresentam as diferenças entre as médias e desvios padrão para os testes da WJ-III, considerando a variável série escolar para os grupos sem dificuldades e com dificuldades de aprendizagem.

Como é possível observar, na Tabela 1, os resultados obtidos pelos alunos da $1^{\underline{a}}$ e $2^{\underline{a}}$ séries tenderam ser maiores para o grupo sem dificuldades em todos os testes da WJ III. Os melhores resultados, tanto para a $1^{\underline{a}}$ quanto para a $2^{\mathrm{a}}$ séries foram para os testes que avaliam a Inteligência Cristalizada (Vocabulário), a Recuperação em Longo Prazo (Aprendizagem Visual e Auditiva e Aprendizagem Visual-Auditiva Adiada), o Pensamento 
Viso-Espacial (Relações Espaciais), a Inteligência Fluida (Formação de Conceitos), Rapidez de Processamento (Emparelhamento Visual), e o Processamento Auditivo (Palavras Incompletas). Assim, foi possível observar que as médias dos testes tendem a aumentar na $2^{\underline{a}}$ série, isto é, ao avançar a série escolar possivelmente há um aumento do desempenho em atividades cognitivas.

Vale comentar que na variável sexo, os alunos do grupo sem dificuldades de aprendizagem que freqüentavam a $2^{a}$ série, apresentaram pontuação superior em todos os testes da WJ III. para ambos os sexos, com exceção do Pensamento Viso-Espacial (Relações Espaciais). Já para o grupo com dificuldades de aprendizagem, os alunos da $2^{\text {a }}$ série também apresentaram pontuação superior a da $1^{\text {a }}$ série em todos os testes, para o sexo masculino.

Por outro lado, as alunas da $1^{\text {a }}$ série tiveram pontuação superior a dos meninos nas habilidades de Inteligência Cristalizada, Recuperação em Longo Prazo,
Processamento Auditivo, Raciocínio Fluido, Rapidez de Processamento e Memória de Trabalho. As alunas da $2^{a}$ série apresentaram pontos superiores aos dos meninos nos testes que avaliam as habilidades Processamento Auditivo, Inteligência Fluida, Raciocínio Fluido, Rapidez de Processamento e Memória de Trabalho Auditiva.

A fim de observar se as diferenças foram significativas entre os grupos, realizou-se a análise multivariada de variância, sendo possível verificar pelo índice Wilk's Lambda efeitos significativos de tipo grupo $(F=4,004$; $p \leq 0,001)$ e série $(F=2,035 ; p \leq 0,05)$ nos testes da Bateria Woodcock-Johnson III. A partir destes resultados, foi feita a análise de variância univariada para analisar cada um dos testes da Bateria WJ III segundo estas variáveis, sendo estes resultados apresentados na Tabela 2.

A Tabela 2, a seguir, apresenta a análise de variância (ANOVA) para os testes da WJ-III.

Tabela 1. Médias e desvios padrão dos testes da bateria WJ-III dos grupos sem dificuldade e com dificuldade de aprendizagem de acordo com a série escolar.

\begin{tabular}{|c|c|c|c|c|c|c|c|c|}
\hline \multirow{3}{*}{ Testes } & \multicolumn{4}{|c|}{ Sem dificuldade } & \multicolumn{4}{|c|}{ Com dificuldade } \\
\hline & \multicolumn{2}{|c|}{$1^{a}$ serie } & \multicolumn{2}{|c|}{$2^{a}$ serie } & \multicolumn{2}{|c|}{$1^{a}$ serie } & \multicolumn{2}{|c|}{$2^{\mathrm{a}}$ serie } \\
\hline & Média & DP & Média & DP & Média & DP & Média & DP \\
\hline $1 \mathrm{~A}$ & 25,40 & 5,85 & 30,60 & 6,49 & 19,24 & 3,25 & 23,15 & 6,97 \\
\hline $1 \mathrm{~B}$ & 2,00 & 2,05 & 4,45 & 2,23 & 1,35 & 0,99 & 1,46 & 0,96 \\
\hline $1 \mathrm{C}$ & 6,30 & 3,12 & 9,25 & 1,55 & 4,88 & 2,87 & 5,92 & 3,06 \\
\hline $1 \mathrm{D}$ & 5,80 & 2,74 & 10,55 & 3,12 & 2,76 & 1,98 & 5,00 & 2,73 \\
\hline 2 & 71,20 & 5,84 & 76,80 & 7,26 & 56,29 & 11,81 & 64,69 & 13,34 \\
\hline 3 & 56,80 & 4,70 & 57,25 & 5,60 & 49,41 & 5,84 & 54,69 & 5,31 \\
\hline 4 & 14,50 & 2,91 & 15,45 & 2,92 & 12,76 & 3,38 & 13,00 & 2,44 \\
\hline 5 & 15,50 & 4,40 & 22,95 & 4,40 & 14,12 & 5,98 & 14,92 & 3,94 \\
\hline 6 & 18,70 & 3,46 & 25,80 & 2,56 & 15,00 & 4,51 & 21,23 & 3,74 \\
\hline 7 & 7,10 & 1,59 & 9,10 & 1,99 & 4,65 & 2,57 & 6,54 & 2,69 \\
\hline 8 & 12,30 & 5,39 & 14,95 & 3,12 & 8,88 & 4,82 & 11,92 & 4,13 \\
\hline $9 \mathrm{P}$ & 5,70 & 3,65 & 9,70 & 2,15 & 4,24 & 2,46 & 5,15 & 3,02 \\
\hline $9 \mathrm{~N}$ & 2,90 & 2,07 & 5,65 & 2,18 & 2,76 & 1,67 & 2,92 & 1,80 \\
\hline 10 & 76,70 & 10,54 & 79,75 & 9,40 & 55,29 & 10,51 & 62,92 & 11,96 \\
\hline
\end{tabular}

1 A - Vocabulário; 1 B - Sinônimos; 1 C - Antônimos; 1 D - Analogias; 2 - Aprendizagem Visual Auditiva; 3 - Relações Espaciais; 4 Combinação de Sons; 5 - Formação de Conceitos; 6 - Rapidez de Processamento; 7 - Números Invertidos; 8 - Palavras Incompletas; 9 P Memória Auditiva Palavras; 9 N - Memória Auditiva Números; 10 - Aprendizagem Visual Auditiva Adiada 
Tabela 2. Análise de Variância para a Bateria WJ-III de acordo com grupo, sexo e série escolar.

\begin{tabular}{|c|c|c|c|c|c|c|c|c|}
\hline Testes & Idade & Grupo & Sexo & Série & $\begin{array}{c}\text { Grupo x } \\
\text { Sexo }\end{array}$ & $\begin{array}{c}\text { Grupo x } \\
\text { Série }\end{array}$ & $\begin{array}{c}\text { Sexo x } \\
\text { Série }\end{array}$ & $\begin{array}{c}\text { Grupo x } \\
\text { Sexo x } \\
\text { Série }\end{array}$ \\
\hline & $\mathrm{F}$ & $\mathrm{F}$ & $\mathrm{F}$ & $\mathrm{F}$ & $\mathrm{F}$ & $\mathrm{F}$ & $\mathrm{F}$ & $\mathrm{F}$ \\
\hline $1 \mathrm{~A}$ & 0,657 & $31,937 * * *$ & $8,462 * *$ & 0,913 & 0,001 & 2,305 & 4,379 & 0.611 \\
\hline $1 \mathrm{~B}$ & 0,76 & $13,961 * * *$ & 0,008 & 2,380 & 0,520 & 7,305 & 1,417 & 0,675 \\
\hline $1 \mathrm{C}$ & 0,003 & $11,168 * *$ & 0,998 & 2,402 & 0,277 & 2,363 & 0,356 & 0,000 \\
\hline $1 \mathrm{D}$ & 0,234 & $33,672 * * *$ & 0,131 & 7,367 & 1,567 & 3,890 & 2,043 & 0,000 \\
\hline 2 & 2,343 & $21,308 * * *$ & 0,577 & $6,092 *$ & 0,189 & 0,217 & 1,980 & 2,802 \\
\hline 3 & 0,433 & $10,838 * *$ & 2,181 & 1,912 & 0.052 & 1,956 & 0,000 & 1,190 \\
\hline 4 & 0,399 & $4,174 *$ & 2,596 & 4,831 & 0,928 & 0,033 & 1,271 & 0,696 \\
\hline 5 & 0,121 & $4,506 *$ & 0,094 & 0,466 & 0,222 & $0,025 *$ & 0,091 & 0,404 \\
\hline 6 & 0,424 & $8,668 * * *$ & 0,067 & $5,972 * * *$ & 0,052 & 4,463 & 0,272 & 0,000 \\
\hline 7 & 0,159 & $12,003 * * *$ & 0,708 & $20,131 * *$ & 0,011 & 0,046 & 0,016 & 0,120 \\
\hline 8 & 0,46 & $13,842 *$ & 1,111 & $3,704 *$ & 0,011 & 5,012 & 2,617 & 0,015 \\
\hline $9 \mathrm{P}$ & 0,625 & $6,113 *$ & 0,606 & 1,289 & 0,178 & $5,123 *$ & 0,002 & 0,050 \\
\hline $9 \mathrm{~N}$ & 1,137 & $42,906 *$ & 1,493 & 2,317 & 2,186 & $0,186 *$ & 0,974 & 2,251 \\
\hline 10 & 2,371 & $13,467 * * *$ & 3,051 & 9,070 & 1,171 & 0,079 & 0,011 & 0,511 \\
\hline
\end{tabular}

$* \mathrm{p} \leq 0,05 ; * * \mathrm{p} \leq 0,01 ; * * * \mathrm{p} \leq 0,001$

1 A - Vocabulário; 1 B - Sinônimos; 1 C - Antônimos; 1 D - Analogias; 2 - Aprendizagem Visual Auditiva; 3 - Relações Espaciais; 4 Combinação de Sons; 5 - Formação de Conceitos; 6 - Rapidez de Processamento; 7 - Números Invertidos; 8 - Palavras Incompletas; 9 P Memória Auditiva Palavras; 9 N - Memória Auditiva Números; 10 - Aprendizagem Visual Auditiva Adiada

A análise dos dados demonstrou que o efeito grupo (sem e com dificuldade de aprendizagem) influenciou significativamente todos os testes das habilidades cognitivas mensuradas pela WJ III. Deste modo pode-se afirmar que há diferenças entre as habilidades cognitivas de crianças sem e com dificuldades de aprendizagem. Nos testes que avaliam o Raciocínio Fluido (Formação de Conceitos) e a Memória de Trabalho (Memória Auditiva Palavras e Memória Auditiva Números), observou-se interação entre as variáveis grupo e série escolar. Isto significa que quando a variável dificuldade de aprendizagem estiver presente, a variável série escolar deverá ser levada em consideração para estes testes. Deve-se destacar que para os testes que medem Recuperação em Longo Prazo (Aprendizagem Visual Auditiva), Rapidez de Processamento (Emparelhamento Visual), Memória de Trabalho (Números Invertidos), e Processamento Auditivo (Palavras Incompletas), a variável série escolar influencia significativamente o desempenho dos participantes.
No teste vocabulário, houve a influência significativa do sexo $(p \leq 0,01)$. Ao se observar as médias obtidas pelo sexo tanto para o grupo sem dificuldades quanto para 0 grupo com dificuldades de aprendizagem, 0 sexo masculino obteve pontuação superior ao feminino nas duas séries escolares neste teste.

Outro ponto investigado neste estudo foi a relação entre notas escolares de crianças com dificuldades de aprendizagem e crianças sem dificuldades de aprendizagem. Vale lembrar que o sistema de avaliação das escolas expressa o rendimento acadêmico por meio de conceitos. Portanto, para a realização deste estudo os conceitos foram convertidos em notas, como já explicados anteriormente. As médias das notas escolares em Português $(M=9,16)$, Matemática $(M=9,5)$ e Ciências $(M=9,66)$ foram superiores para o grupo sem dificuldade de aprendizagem, quando comparadas às do grupo com dificuldade de aprendizagem, Português $(M=5,4)$, Matemática $(M=5,71)$ e Ciências $(M=6,35)$. 
Tabela 3. Correlação de Pearson entre notas escolares e Testes da WJ-III de acordo com a dificuldade de aprendizagem.

\begin{tabular}{|c|c|c|c|c|c|c|}
\hline \multirow{2}{*}{ Testes } & \multicolumn{3}{|c|}{ Sem dificuldade } & \multicolumn{3}{|c|}{ Com dificuldade } \\
\hline & Português & Matemática & Ciências & Português & Matemática & Ciências \\
\hline $1 \mathrm{~A}$ & $0,391^{*}$ & 0,233 & 0,240 & 0,039 & 0,017 & 0,053 \\
\hline $1 \mathrm{~B}$ & 0,183 & 0,060 & $-0,057$ & $0,374 *$ & 0,243 & 0,214 \\
\hline $1 \mathrm{C}$ & $0,440^{*}$ & 0,049 & 0,196 & $0,390 *$ & 0,181 & 0,134 \\
\hline $1 \mathrm{D}$ & $0,413^{*}$ & 0,096 & 0,261 & 0,210 & 0,042 & 0,203 \\
\hline 2 & $0,383^{*}$ & 0,273 & 0,211 & 0,245 & 0,145 & 0,262 \\
\hline 3 & 0,043 & $-0,213$ & $-0,119$ & 0,084 & $-0,054$ & $-0,022$ \\
\hline 4 & $0,448^{*}$ & 0,289 & $0,365^{*}$ & 0,308 & 0,135 & 0,287 \\
\hline 5 & $0,512 * *$ & 0,262 & 0,276 & $0,456^{*}$ & 0,241 & 0,288 \\
\hline 6 & 0,230 & $-0,081$ & 0,063 & 0,220 & 0,077 & 0,224 \\
\hline 7 & 0,214 & 0,051 & 0,159 & 0,342 & 0,206 & 0,298 \\
\hline 8 & $0,449^{*}$ & 0,316 & 0,261 & $0,417 *$ & 0,248 & 0,284 \\
\hline $9 \mathrm{P}$ & $0,475 * *$ & 0,338 & 0,342 & $0,369 *$ & 0,134 & 0,262 \\
\hline $9 \mathrm{~N}$ & $0,594 * *$ & $0,376^{*}$ & 0,264 & 0,340 & 0,102 & 0,287 \\
\hline 10 & $0,576^{* *}$ & 0,320 & 0,077 & 0,121 & 0,059 & 0,187 \\
\hline
\end{tabular}

$* \mathrm{p} \leq 0,05 ; * \mathrm{p}^{\mathrm{s}} \leq 0,01$

Nota: 1 A - Vocabulário; 1 B - Sinônimos; 1 C - Antônimos; 1 D - Analogias; 2 - Aprendizagem Visual Auditiva; 3 - Relações Espaciais; 4 - Combinação de Sons; 5 - Formação de Conceitos; 6 - Rapidez de Processamento; 7 - Números Invertidos; 8 - Palavras Incompletas; 9 P - Memória Auditiva Palavras; 9 N - Memória Auditiva Números; 10 - Aprendizagem Visual Auditiva Adiada

Os resultados, na Tabela 3, mostram as correlações de Pearson entre as notas escolares e a pontuação média obtida na WJ-III para os alunos de acordo com o grau de dificuldade de aprendizagem.

Os resultados descritos nesta tabela indicaram correlações positivas significativas entre as notas escolares e os testes da WJ-III no grupo de crianças sem dificuldades de aprendizagem. A disciplina Português apresentou correlação significativa e positiva com as habilidades Inteligência Cristalizada (Vocabulário), Raciocínio Fluido (Formação de Conceitos), Memória de Trabalho (Memória Auditiva por palavras e Memória Auditiva por Números) e Recuperação em Longo Prazo (Aprendizagem Auditiva Visual Adiada).

Importante sinalizar que, a disciplina Português não apresentou relação com as habilidades Pensamento VisoEspacial (Relações Espaciais), Rapidez de Processamento (Emparelhamento Visual), Memória de Trabalho (Números Invertidos). A disciplina Ciências correlacionou apenas com a Memória Auditiva (Combinação de sons). Entretanto, neste grupo houve correlações significativas entre as notas da disciplina Matemática e a habilidade Memória de Trabalho (Memória Auditiva por Números).

Quanto ao grupo com dificuldades de aprendizagem notaram-se correlações significativas positivas dos testes de inteligência da Bateria WJ-III entre as notas da disciplina Português e as habilidades de Inteligência Cristalizada (Sinônimos e Antônimos), Raciocínio Fluido (Formação de Conceitos), Processamento Auditivo (Palavras Incompletas), e Memória de Trabalho (Memória Auditiva Palavras). Não houve correlação significativa entre os resultados da WJ-III e as notas nas disciplinas Matemática e Ciências.

\section{Discussão}

O propósito da presente pesquisa foi avaliar as habilidades cognitivas de crianças de $1^{\underline{a}}$ e $2^{\underline{a}}$ séries do Ensino Fundamental. A partir de tal estudo, foi possível utilizar um instrumento que está sendo validado para a população brasileira, a Bateria de Habilidades Cognitivas Woodcock-Johnson III, McGrew, K.S., Woodcock, R.W. \& Mather, N. (2001) em dois grupos de crianças: sem indicação de dificuldades de aprendizagem e crianças com indicação de dificuldades de aprendizagem.

Os resultados demonstraram ser possível confirmar a hipótese de que há diferença nas habilidades cognitivas avaliadas pela bateria WJ III para o grupo com dificuldade de aprendizagem e o grupo sem dificuldades de aprendizagem. O desempenho do grupo com dificuldades de aprendizagem foi claramente menor em todos os testes da bateria WJ-III. Desta forma, confirmou-se a validade dessa bateria para identificar crianças com dificuldades de aprendizagem e sua relevância no contexto educacional, pois permitirá ao professor conhecer do aluno as habilidades que mais necessitam ser estimuladas.

Um dos modelos mais utilizados para definir as dificuldades de aprendizagem trata-se do critério de discrepância entre o desempenho em testes que avaliam as habilidades cognitivas do sujeito e o seu desempenho acadêmico em provas que avaliam leitura, escrita e 
matemática (Dobrowskii \& cols., 2004). Stanford e Oakland (2000) afirmam que uma definição comum sobre dificuldades de aprendizagem favorecerá 0 desenvolvimento de instrumentos que possam avaliar tais dificuldades.

Reafirma-se, portanto, a necessidade de instrumentos que avaliem diferentes habilidades, como a bateria Woodcock-Johnson III, pois permitem delinear um perfil mais diversificado das habilidades cognitivas da criança identificando as habilidades que estão mais deficitárias. No entanto, há escassez de pesquisas, no Brasil, com instrumentos validados que investiguem as características cognitivas de crianças com dificuldades de aprendizagem para que, posteriormente, possam ser desenvolvidos programas de intervenção escolar que auxiliem essas crianças na superação das dificuldades de aprendizagem. O Conselho Federal de Psicologia (2004) tem se posicionado de modo mais crítico em relação à qualidade técnico-científica dos instrumentos psicológicos, criando procedimentos para verificar os padrões de validade e precisão dos testes psicológicos.

Em relação a variável sexo, observou-se forte influência no subteste Vocabulário, que é uma medida de inteligência cristalizada. Nesse subteste, predominaram médias mais altas para o sexo masculino, tanto para 0 grupo sem dificuldades de aprendizagem, quanto para o grupo com dificuldades de aprendizagem. Este resultado corrobora os trabalhos de Wechsler e cols. (2007), que também observaram desempenho superior do sexo masculino na habilidade de Inteligência Cristalizada.

Outra informação interessante refere a variável série escolar. Há uma tendência em aumentar o desempenho dos testes à medida que a criança avança em tempo de escolaridade, principalmente, aqueles que avaliam recuperação a longo prazo (testes 2 e 10 ) e processamento auditivo (teste 6 e 8 ), memória de trabalho (testes 7 e 9) e Velocidade de Processamento (teste 7) habilidades relacionadas, respectivamente, às habilidades de pensamento e eficiência cognitiva (Munhoz-Sandoval \& Woodcock, 1999). Alves (1998) discutiu a influência de algumas variáveis sobre os resultados dos testes de inteligência. Dentre as variáveis, a autora aponta a série escolar como uma das variáveis significativas nos resultados dos testes de inteligência. Os resultados dos testes tendem a aumentar à medida que a criança avança em idade e em escolaridade.

Outro ponto a ser discutido diz respeito às relações entre as habilidades descritas no modelo $\mathrm{CHC}$ e o rendimento em leitura e Matemática. Flanagan e Ortiz (2001) associam o rendimento em Matemática às seguintes habilidades cognitivas: Raciocínio Fluido, Inteligência Cristalizada, Memória de Trabalho, Velocidade de Processamento, sendo que esta última é importante em todos os anos escolares, principalmente, nos anos iniciais. Por outro lado, o desempenho em Português relaciona-se a Inteligência Cristalizada, Memória de Trabalho, Processamento auditivo, Recuperação em longo prazo e Velocidade de processamento. Nos anos iniciais da escolarização, período em que a criança inicia a aprendizagem da leitura predominam as habilidades de Processamento Auditivo, Recuperação em Longo Prazo e Velocidade de Processamento.

Nos resultados dessa pesquisa foi possível verificar correlações significativas e positivas no grupo sem dificuldades entre as habilidades de Inteligência
Cristalizada, Raciocínio Fluido, Processamento Auditivo, Memória de Trabalho Auditiva e Recuperação em Longo Prazo e as notas da disciplina Português. No entanto, o grupo com dificuldades associou-se de forma significante e positiva, apenas entre as habilidades Inteligência Cristalizada, Raciocínio Fluido, Processamento Auditivo, Memória de Trabalho Auditiva e as notas da disciplina Português. Neste caso, devem ser verificadas quais habilidades são trabalhadas pelos conteúdos escolares, assim como as estratégias utilizadas pelos professores, pois a avaliação na sala de aula deve estar relacionada à proposta de trabalho da escola e ao perfil de aluno que se quer formar (Souza, 2000). Como pode ser notada neste estudo, a disciplina Português é prioritária nas duas séries pesquisadas e provavelmente 0 insucesso da criança nesta disciplina compromete o seu desempenho escolar. Valente (2003) alerta que, a avaliação na escola deve assumir um caráter contínuo para realmente acompanhar o desenvolvimento dos alunos.

Outro ponto investigado foi a relação entre desempenho acadêmico e os resultados obtidos nos testes da WJ III. Esses resultados permitiram observar que os testes de ambos os grupos tiveram maior relação com a nota da disciplina Português. Sobre este aspecto foi possível constatar que nas séries iniciais do Ensino Fundamental, focalizam-se mais os conteúdos relacionados ao aprendizado da leitura e da escrita. Nessa direção Curi (2002) comenta que a aquisição da leitura e da escrita requer esforço, sendo a escola responsável pela introdução da criança no mundo da escrita e da leitura. $\mathrm{Na}$ primeira e segunda série o objetivo principal é a alfabetização dos alunos. Os testes da WJ III mais relacionados ao desempenho escolar de Português foram aqueles que mediam habilidades relacionadas à Inteligência Cristalizada (Gc), Inteligência Fluida (Gf), Memória a Curto Prazo (Gsm), Armazenamento e Recuperação Associativa a Longo Prazo (Glr). Essas habilidades gerais são mais relacionadas ao desempenho acadêmico, como afirma Schelini (2002).

De modo geral, esta pesquisa suscitou algumas ponderações em relação às dificuldades de aprendizagem que a criança vivencia no início do processo de escolarização. $O$ desempenho acadêmico nas séries iniciais focaliza a aprendizagem da leitura e da escrita. As pesquisas realizadas sobre dificuldades de aprendizagem estão, em sua maioria, associadas à leitura, à escrita e à Matemática. A disciplina Português tem caráter prioritário, no início de escolarização, em que há destaque para o processo de alfabetização. As dificuldades no processo de alfabetização favorecem o aumento do fracasso escolar.

Os dados obtidos na bateria WJ III sugerem que esta é válida, pois consegue distinguir crianças com e sem dificuldades de aprendizagem. É possível, por meio desta bateria, identificar crianças que apresentem dificuldades em diferentes habilidades cognitivas, principalmente aquelas relacionadas com o desempenho acadêmico.

Por outro lado, seria interessante e, ao mesmo tempo necessário, comparar as habilidades cognitivas deficitárias com a avaliação da área médica, como déficit de atenção, visão, audição. Pois um dos critérios para identificar a criança com dificuldade de aprendizagem é a eliminação de alterações sensoriais.

Assim, as contribuições desta pesquisa estendem-se para os psicólogos escolares brasileiros, que necessitam orientar os professores para identificar e intervir 
adequadamente junto a alunos que estejam apresentando dificuldades de aprendizagem.

\section{Referências}

Alves, I. B. (1998). Variáveis significativas na avaliação da inteligência. Psicologia Escolar e Educacional, 2(2), 109-114.

Azevedo, A. C. P. (2002). Brinquedoteca como estratégia de diagnóstico e intervenção em dificuldades escolares. Tese de Doutorado, Pontifícia Universidade Católica de Campinas, Campinas, São Paulo.

Bispo, N. L. (2000). Imagem mental, memória e dificuldades de aprendizagem na escrita. Dissertação de Mestrado, Universidade Estadual de Campinas, Campinas, São Paulo.

Boarini, M. L., \& Borges, R. F. (1998). Demanda infantil por serviços de saúde mental: sinal de crise. Estudos de Psicologia, 3(1), 83-108.

Cabral, E., \& Sawaia, S. M. (2001). Concepções e atuação profissional diante das queixas escolares: os psicólogos nos serviços públicos de saúde. Estudos de Psicologia, 6(2), 143-155.

Capovilla, A. G., Güstchow, C. R. D., \& Capovilla, F. C. (2004). Habilidades de leitura que predizem competência de leitura e escrita. Psicologia: Teoria e Prática, 6(2), 13-23.

Carneiro, G. R. S. (2002). O autoconceito de crianças com dificuldade de aprendizagem na escrita. Dissertação de Mestrado, Universidade Estadual de Campinas, Campinas, São Paulo.

Ciasca, S. M. (2003). Distúrbios e dificuldades de aprendizagem: questão de nomenclatura. Em S. M. Ciasca (Org.), Distúrbios de aprendizagem: proposta de avaliação interdisciplinar (pp. 19-31). São Paulo: Casa do Psicólogo.

Coll, L. R. (2000). Revendo a atuação do psicólogo escolar com crianças que apresentam dificuldades no processo de aquisição da leitura e da escrita. Expressão, 4(1), 91-102.

Conselho Federal de Psicologia (2004). Testes Psicológicos. Suplemento especial. Ano XVIII, 77(Fevereiro). Brasília: DF, Conselho Federal de Psicologia.

Corsini, C. F. (1998). Dificuldade de aprendizagem: representações sociais de professores e alunos. Dissertação de Mestrado, Pontifícia Universidade Católica de Campinas, Campinas, São Paulo.

Curi, N. M. (2002). Atenção, Memória e Dificuldades de Aprendizagem. Tese de Doutorado, Universidade Estadual de Campinas, Campinas, São Paulo.

Dombrowski, S. C., Reynolds, C. R., \& Kamphaus, R. W. (2004). After demise of the discrepancy: proposed learning disabilities diagnostic criteria. Professional psychology Research and Pratic, 4, 364-372.

Flanagan, D. P., McGrew, K. S., \& Ortiz, S. O. (2000). The Wechsler Intelligence Scales and Gf-Gc theory: a contemporany approach to interpretation. Boston: Allyn and Bacon.

Flanagan, D., \& Ortiz, S. (2001). Essentials of Cross-Battery Assessment. New York: John Wiley and Sons.
Kaminski, R. A., \& Good, R. (1996). Toward a techonology for assessing basic early literacy skills. School Psychology Rewiew, 25(2), 215-227.

Kennard, B. D., Stewart, S. M., Silver, C. H., \& Emslie, G. J. (2000). Neuropsyychological ablities and academic gains in learning disabled children. School Psychology International, 21(2), 172-176.

McGrew, K. S., \& Flanagan, D. P. (1998). The intelligence test desk reference (ITDR) - Gf-Gc cross battery assessment. Boston: Allyn and Bacon.

McGrew, K.S., Woodcock, R.W., \& Mather, N. (2001). WoodcockJohnson III: Tecnical Manual. Itasca, II: Riverside Publishing.

Muñoz-Sandoval, A., \& Woodcock, R. W. (1999). Batería Woodcock-Muñoz: Pruebas de Habilidade CognitivaRevisada/Bateria Woodcock-Muñoz: Pruebas de Aprovechamiento - Revisada. Em S. M. Wechsler \& R. S. L. Guzzo (Orgs.), Avaliação psicológica: Perspectiva internacional (pp. 261-297). São Paulo: Casa do Psicólogo.

Rossini, S. D. R., \& Santos, A. A. A. (2002). Fracasso escolar: estudo documental de encaminhamentos. Em F. F. Sisto, E. Boruchovitch, L. D. T. Fini, R. P. Brenelli \& S. C. Martinelli (Orgs.), Dificuldades de aprendizagem no contexto psicopedagógico (pp. 214-235). Petrópolis: Vozes.

Santos, M. A., \& Primi, R. (2005). Desenvolvimento de um teste informatizado para avaliação do raciocínio, da memória e da velocidade do processamento. Estudos de Psicologia, 22(3), 241254.

Saravali, E. G. (2003). Dificuldades de aprendizagem e interação social. Tese de Doutorado, Universidade Estadual de Campinas, Campinas, São Paulo.

Schelini, P. W. (2002). Bateria multidimensional de inteligência infantil: proposta de instrumento. Tese de Doutorado, Pontifícia Universidade Católica de Campinas, Campinas, São Paulo.

Sisto, F. F. (2002). Dificuldades de aprendizagem. Em F. F. Sisto, E. Boruchovitch, L. D. T. Fini, R. P. Brenelli \& S. C. Martinelli (Orgs.), Dificuldades de aprendizagem no contexto psicopedagógico (pp. 19-39). Petrópolis: Vozes.

Souza, C. P. (2000). Dimensões da avaliação educacional. Estudos em Avaliação Educacional, 22, 101-118.

Stanford, G., \& Oakland, T. (2000). Cognitive deficits underlying learning disabilities. School Psychology International, 21(3), 306321.

Valente, S. M. P. (2003). A avaliação da aprendizagem no contexto da reforma educacional brasileira. Estudos em Avaliação educacional, 28, 75-88.

Wechsler, S. M., Ferreira, A., Pereira, D. P., Mundin, M. C., Coraini, B., \& Prette, P. (2007). Habilidades cognitivas e rendimento escolar em estudantes brasileiros. Submetido à publicação.

Wechsler, S. M., \& Schelini, P. W. (2006). Bateria de habilidades cognitivas Woodcock-Johnson III: validade de construto. Psicologia: teoria e pesquisa, 22(3), 287-295. 
Wechsler, S. M., Vendramini, C. M., \& Schelini, P. W. (2007). Adaptação brasileira dos testes verbais da Bateria WoodcockJohnson III. Revista Interamericana de Psicologia, 41, 285-294.

Woodcock, R. W., McGrew, K., \& Mather, N. (2001). Technical Manual. Woodcock-Johnson III. Itasca, IL: Riverside Publishing.
Zucoloto, K. A. (2001). A compreensão da leitura em crianças com dificuldades na escrita. Dissertação de Mestrado, Universidade Estadual de Campinas, Campinas, São Paulo.

Zucoloto, K. A., \& Sisto, F. F. (2002). Dificuldades de aprendizagem em escrita e compreensão em leitura. Interação em Psicologia, 6(2), 157-166.

Recebido em: 12/06/2007

Revisado em: 30/04/2008

Aprovado em: 27/08/2008

\section{Sobre as autoras}

Dalva Alice Rocha Mól (molrangel@uol.com.br) - Faculdades Integradas de Santa Fé do Sul Rua 18, no 1290 , Centro

15775-000 Santa Fé do Sul - SP

Solange Muglia Wechsler (wechsler@lexxa.com.br) - Pontifícia Universidade Católica de Campinas. Psicologia, Campus II, Pontifícia Universidade Católica de Campinas

Av. John Boyde Dunlop, s/n Jardim Ipaussurama

13060-904 - Campinas - SP 
